\title{
A Novel Pulse Coupled Neural Network Based Method for Multi-focus Image Fusion
}

\author{
Yongxin Zhang ${ }^{1,2}$, Li Chen ${ }^{1}$, Zhihua Zhao ${ }^{1}$ and Jian Jia ${ }^{3}$ \\ ${ }^{1}$ School of Information Science and Technology, Northwest University, Xi'an, \\ 710127, China \\ ${ }^{2}$ Luoyang Normal University, Luoyang, 471022, China \\ ${ }^{3}$ Department of Mathematics, Northwest University, Xi'an, 710127, China \\ tabo126@126.com,chenli@nwu.edu.cn
}

\begin{abstract}
Multi-focus image fusion means to fuse multiple source images with different focus settings into one image, so that the resulting image appears sharper. In order to extract the focused regions of the fused image efficiently, a novel pulse coupled neural network (PCNN) method for multi-focus image fusion is proposed. The registered source images are decomposed into principal components and sparse components by robust principal component analysis (RPCA) decomposition, and the important features of the sparse components are used to motivate the PCNN neurons, whose outputs detect the focused regions of the source images and integrate them to construct the final fused image. Experimental results show that the proposed scheme works better in extracting the focused regions and improving the fusion quality compared to the other existing fusion methods in terms of mutual information (MI) and $Q^{A B / F}$.
\end{abstract}

Keywords: image fusion, pulse coupled neural network, blocking artifacts, sparse feature

\section{Introduction}

For the limited depth-of-field of optical lenses in imaging sensors, it is impossible to obtain an image where all objects appear sharp. The objects within the depth of field are sharp and the objects out of the depth of field are blurred. Multi-focus image fusion is an effective way to extend the depth of the field. In general, image fusion methods can be categorized into two groups: spatial domain fusion and transform domain fusion [1]. The spatial domain fusion methods are easy to implement and have low computational complexity, while the spatial domain methods may produce blocking artifacts and compromise the quality of the final fused image. Different from the spatial domain fusion, the transform domain fusion methods can get improved contrast, as well as signal-to-noise ratio and better fusion quality [2], while the transform domain fusion methods are more time/space-consuming to implement.

Pulse coupled neural network (PCNN) is a novel visual cortex-inspired neural network developed by Eckhorn, et al., [3]. Broussard, et al., [4] have firstly applied PCNN in image fusion for object detection and Johnson, et al., [5] have pointed out the great potential of PCNN in the field of data fusion in the same year. It has been observed that PCNN based image fusion methods outperform the conventional methods [6]. So far, many multi-focus image fusion methods based on PCNN have been proposed [7-9]. However, most of them suffer from various problems. Miao, et al., [7] have proposed a fusion method using the sharpness of a small neighborhood of each pixel as the linking strength of PCNN. It works well in preserving edge and texture information, but suffers from contrast reduction. Huang, 
et al., [8] have proposed a fast fusion method based on energy of the image laplacian (EOL) motivated PCNN in spatial domain. It produces the blocking artifacts in fused image while improves the fusion speed. Qu, et al., [9] have developed a fusion method based on spatial frequency (SF) motivated PCNN in nonsubsumpled contourlet transform (NSCT) domain. It works well for multi-focus image and visible/infrared image, but the absence of directional information in SF and the use of the same fusion rule for all the sub-bands lead to the contrast reduction and the loss of image details.

Different from the fusion methods mentioned above, a new PCNN based image fusion method is proposed. Robust principal component analysis (RPCA) is an important method of low-rank matrix recovery [10], which decomposes an image into a low-rank matrix which corresponds to the background, and a sparse one which links to salient objects [11]. Wan, et al., [12] have investigated the potential application of RPCA in the multi-focus image fusion and achieved a consistently good fusion result, but their method requires longer computational time. Different from Wan's method, this paper use the salient features of the sparse component to motivate the PCNN neurons for image fusion. The feature maps of the sparse componets are used to motivate the PCNN neurons. The focused regions are detected by comparing the firing times of the PCNN neurons. The proposed method can efficiently extract the focused regions details from the source images and improve the visual quality of the fused image.

The rest of the paper is organized as follows. In Section 2, the basic concept of PCNN will be briefly described, followed by the new method with PCNN for image fusion in Section 3. In Section 4, extensive simulations are performed to evaluate the performance of the proposed method. In addition, several experimental results are presented and discussed. Finally, concluding remarks are drawn in Section 5.

\section{Pulse Coupled Neural Network}

PCNN belongs to the third generation artificial neural network. In image processing, the PCNN is a single layered, two-dimensional, laterally connected with image pixels each other. Each PCNN neuron consists of three parts: receptive field, modulation field and pulse generator. The neuron can be described as Equation (1).

$$
\left\{\begin{array}{l}
F_{i j}(n)=e^{-\alpha_{F}} F_{i j}(n-1)+S_{i j}+V_{F} \sum_{k l} M_{i j k l} Y_{k l}(n-1) \\
L_{i j}(n)=e^{-\alpha_{L}} L_{i j}(n-1)+V_{L} \sum_{k l} W_{i j k l} Y_{k l}(n-1) \\
U_{i j}(n)=F_{i j}(n)\left[1+\beta L_{i j}(n)\right] \\
Y_{i j}(n)= \begin{cases}1 & U_{i j}(n)>\theta_{i j}(n) \\
0 & \text { otherwise }\end{cases} \\
\theta_{i j}(n)=e^{-\alpha_{\theta}} \theta_{i j}(n-1)+V_{\theta} Y_{i j}(n-1)
\end{array}\right.
$$

The indexes $i$ and $j$ refer to the pixel location in the image, $k$ and $l$ refer to the dislocation in a symmetric neighborhood around the one pixel. $n$ denotes the current iteration and $S_{i j}$ denotes the input stimulus such as the normalized gray level of image pixels. $\alpha_{F}, \alpha_{L}$ and $\alpha_{\theta}$ are the decay constants of the PCNN neuron. $V_{F}, V_{L}$ and $V_{\theta}$ are the magnitude scaling terms. The constant $\beta$ is the linking strength. $F_{i j}$ is the primary 
input from the neurons receptive fields. $L_{i j}$ is the secondary input of lateral connections with neighboring neurons. The inter-connections $M$ and $W$ are the constant synaptic weight matrices for $F_{i j}$ and $L_{i j}$, respectively. $\theta$ is a dynamic neuron threshold. The neuron will generate pulse when $U_{i j}(n)>\theta_{i j}(n)$. This pulse is also called one firing time. The sum of $Y_{i j}$ in $n$ iteration is called firing times [6], to represent image information, which is defined as Equation (2).

$$
T_{i j}=T_{i j}(n-1)+Y_{i j}(n)
$$

The advantages of PCNN in image fusion are global coupling and pulse synchronization of neurons. In this paper, the firing times of the PCNN neurons are used to detect the focused regions.

\section{Proposed Method}

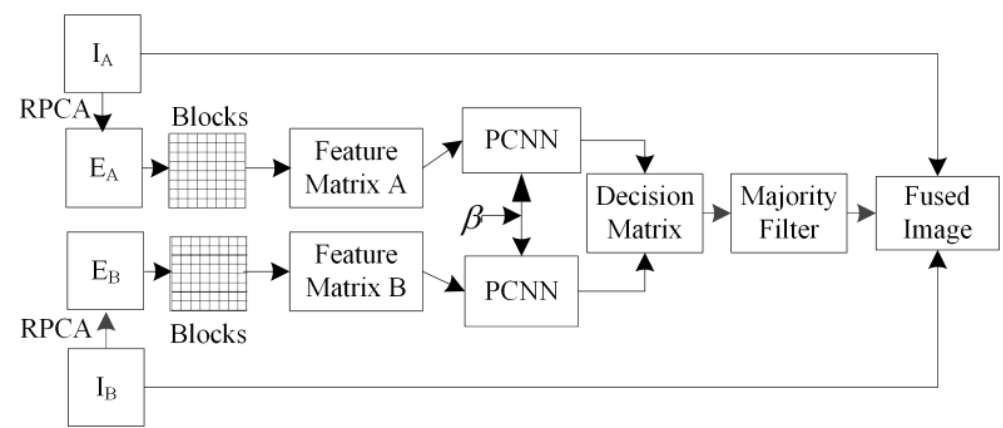

Figure 1. Block Diagram of the Proposed Multi-Focus Image Fusion Framework

In this section, a novel fusion scheme with PCNN is proposed and the fusion framework is depicted in Figure 1. For simplicity, this paper assumes that there are only two source images, namely $I_{A}$ and $I_{B}$. The source images are assumed to preregistered and the image registration is not included in the framework. The fusion algorithm consists of the following five steps:

Step 1: The source images $\left\{I_{A}, I_{B}\right\}, I_{A}, I_{B} \in i^{M \times N}$ is converted into column vectors $I_{A}^{c}, I_{B}^{c} \in \mathrm{i}^{M N \times 1}$, respectively. The data matrix $D$ is defined as:

$$
D=\left[I_{A}^{c} I_{B}^{c}\right]
$$

Step 2: Perform RPCA decomposition on $D$ to obtain a principal matrix $A \in i^{M N \times 2}$ and a sparse matrix $E \in i^{M N \times 2}$, respectively. The sparse matrix $E \in i^{M N \times 2}$ is computed through inexact augmented Lagrange multipliers algorithm (IALM) of RPCA [13], which is a fast version of implementation for recovering low-rank matrices. The sparse matrix $E \in i^{M N \times 2}$ is then converted into matrices $E_{A}, E_{B} \in \mathrm{i}^{M \times N}$ corresponding to the source images $I_{A}$ and $I_{B}$, respectively.

Step 3: Perform block division on $E_{A}, E_{B} \in i^{M \times N}$ to construct feature maps. The sparse matrices $E_{A}$ and $E_{B}$ are divided into blocks with fixed block size, respectively. 
Let $E_{A}^{(k)}$ and $E_{B}^{(k)}$ denote the $k$ th block of the sparse matrices $E_{A}$ and $E_{B}$, respectively. The EOL of each matrix block can be calculated as [14]:

$$
\begin{gathered}
E O L=\sum_{i} \sum_{j}\left(E_{i i}^{2}+E_{j j}^{2}\right) \\
E_{i i}+E_{j j}=-E(i-1, j-1)-4 E(i-1, j)-E(i-1, j+1)-4 E(i, j-1)+20 E(i, j) \\
-4 E(i, j+1)-E(i+1, j-1)-4 E(i+1, j)-E(i+1, j+1)
\end{gathered}
$$

Where $E(i, j)$ indicates the value of the element at the position $(i, j)$ in the sparse matrix block. Let $\operatorname{EOL}_{(k)}^{E_{A}}$ and $\operatorname{EOL}_{(k)}^{E_{B}}$ be the EOL of $E_{A}^{(k)}$ and $E_{B}^{(k)}$, respectively. The EOL of each block of the sparse matrices constructs the feature maps $F_{A}$ and $F_{B}$, respectively.

Step 4: Construct decision matrix by using the output of PCNN. $F_{A}$ and $F_{B}$ are input to PCNN to motivate the neurons to generate pulse with Equation (1), and the firing times are calculated with Equation (2). Thus, a decision matrix $H \in \mathrm{i}^{M \times N}$ is constructed for recording the comparison results according to the selection rule as follows:

$$
H(i, j)=\left\{\begin{array}{cc}
1 & T_{k}^{A}(n) \geq T_{k}^{B}(n) \\
0 & \text { otherwise }
\end{array}\right.
$$

where ' 1 ' in $H$ indicates the pixel $(i, j)$ of the $k$ th block of the source image $I_{A}$ is in focus while ' 0 ' in $H$ indicates the pixel $(i, j)$ of the $k$ th block of the source image $I_{B}$ is in focus.

Step 5: Construct the fused image. A consistency verification process [15] is subsequently applied to refine the decision matrix by using a majority filter, where a pixel is more likely to belong to the label which many of its neighbors also belong. A majority filter [16] is performed on the decision matrix $\mathrm{W}$ to obtain a refined decision matrix. Thus, the final fused image is constructed according to fusion rule as follows:

$$
F(i, j)= \begin{cases}I_{A}(i, j) & H(i, j)=1 \\ I_{B}(i, j) & H(i, j)=0\end{cases}
$$

where $I_{A}(i, j)$ and $I_{B}(i, j)$ are the values of the pixels at position $(i, j)$ in the source images $I_{A}$ and $I_{B}$, respectively. In this paper, the majority filter size is $3 \times 3$.

\section{Experimental Results}

In order to evaluate the performance of the proposed method, several experiments are performed on two pairs of multi-focus source images [17, 18] differing in content and texture, as are shown in Figure 2. The two pairs are all grayscale images with size of $640 \times 480$ pixels. In this paper, all the source images are assumed to have been registered. Experiments are conducted with Matlab in Windows environment on a computer with Intel Xeon X5570 and 48G memory. 


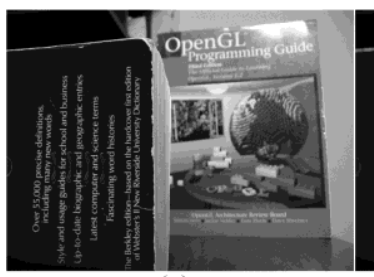

(a)

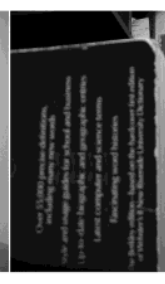

(b)

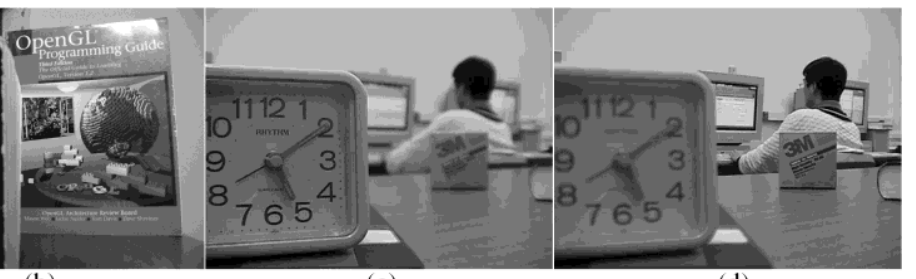

(c) (d)

Figure 2. Multi-focus Source Images. (a) Near Focused Image 'Book', (b) far Focused Image 'Book', (c) Near Focused Image 'Lab', (d) Far Focused Image 'Lab'

For comparison, beside the proposed method, some existing multi-focus image fusion methods are also implemented on the same set of source images. These methods are discrete wavelet transform (DWT), SF (Li's method [19]), PCNN1 (Huang's method [8]), PCNN2 (Miao's method [7]), RPCA (Wan's method [12]). Due to the lack of original source code, this paper uses the Eduardo Fernandez Canga's Matlab image fusion toolbox [20] as a reference for DWT, SF. Specifically, the Daubechies wavelet function 'bi97' is used in the DWT. The decomposition level of DWT is 4. The RPCA toolbox [21] is used as the reference for RPCA decomposition. The PCNN toolbox [22] is used as a reference for PCNN1, PCNN2 and the proposed method. The parameters of PCNN1 are set as $k \times l=13 \times 13, \alpha_{L}=1.0, \alpha_{\theta}=5.0, V_{L}=0.2, V_{\theta}=20.0$ and $N=300$. The parameters of Miao's method are set as $k \times l=3 \times 3, \alpha_{L}=0.9, \alpha_{\theta}=2.5, V_{L}=0.2$, $V_{\theta}=20.0$ and $N=200$. The parameters of the proposed method are set as the same as that of Huang's method and the block size is $8 \times 8$. In order to quantitatively compare the performance of the proposed method and that of the other fusion methods mentioned above, two metrics are used to evaluate the fusion performance. They are: (i) Mutual information (MI) [23], which measures the degree of dependence of the source image and the fused image. (ii) $Q^{A B / F}$ [24], which reflects the amount of edge information transferred from the source images to the fused image. A larger value for them means a better fusion result.

\subsection{Qualitative Analysis}

For visual comparison, the fused images 'Book' and 'Lab' obtained by different methods are shown in Figures 3 (a-f) and Figures 4 (a-f). The difference images between the far focused source image 'Lab' and corresponding fused images obtained by different methods are shown in Figures 5 (a-f). The fused images obtained by the other fusion methods demonstrate obviously blur and artifacts, such as the upper edge and text region of the left books in Figures 4 (a-e), the upper edge of the student's head in Figures 4 (a), (b), (d) and (e). The obvious blocking artifacts appear in the fused image obtained by SF and PCNN1, such as the text region of the left book in Figures 4 (b) and (d), the upper edge of the student's head in Figures 4 (b) and (d). There is a narrow prominent appears on the upper edge of the student's head in Figure 4 (c). There are some obvious residuals in the difference images, such as the left regions in Figures 5 (a-e). The obvious distortion appears in Figure 5 (a). Therefore, the fused image of the proposed method achieves superior visual performance by containing all the focused contents from the source images without introducing artifacts. 


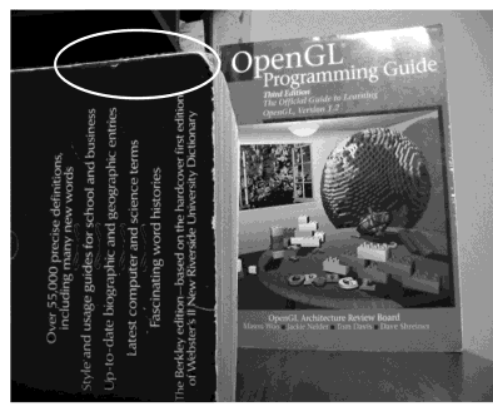

(a)
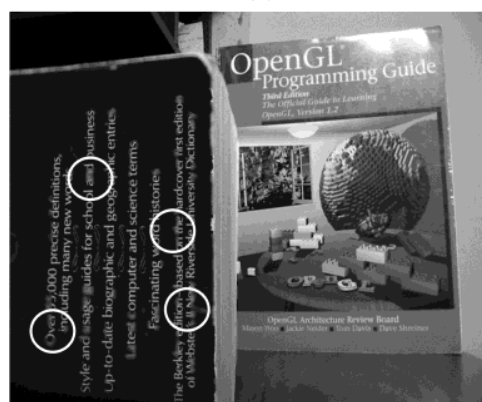

(d)
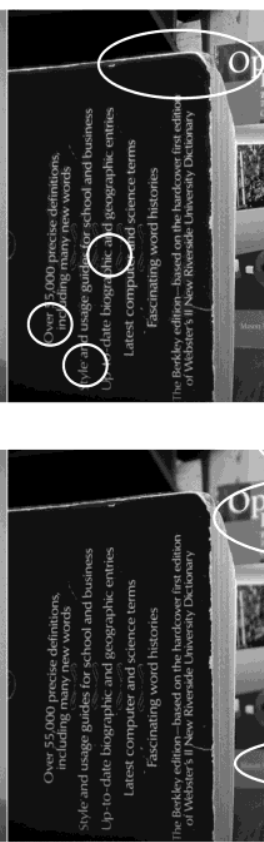

(e)

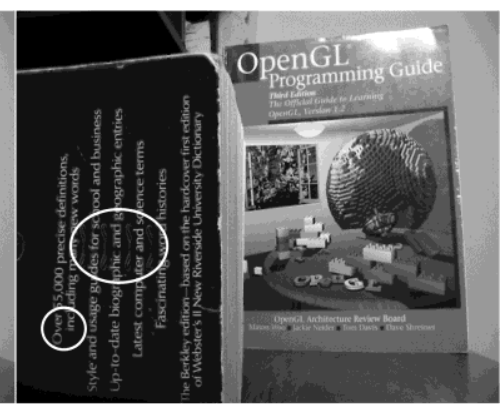

(c)

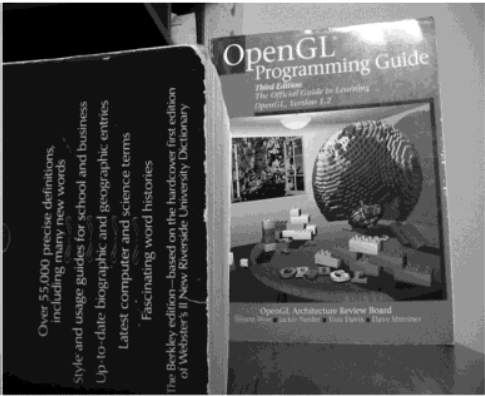

(f)

Figure 3. Fused Images 'Book' Obtained by DWT (a), SF (b), RPCA (c), PCNN1 (d), PCNN2 (e), the Proposed Method (f)

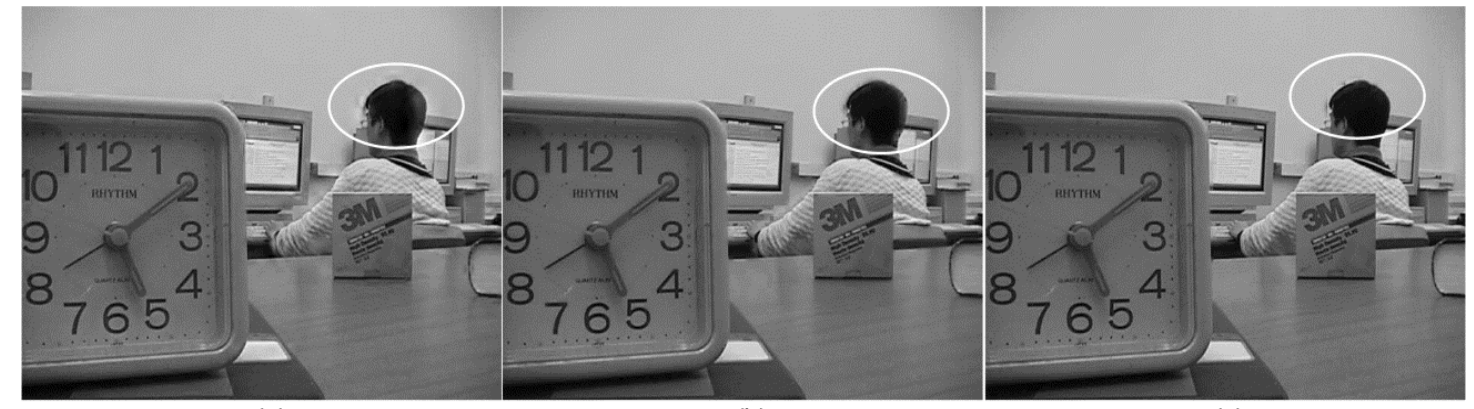

(a)

(b)

(c)

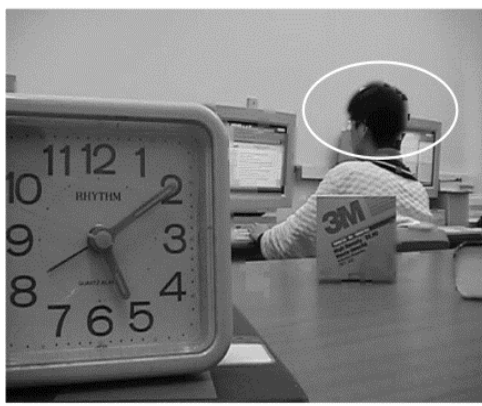

(d)

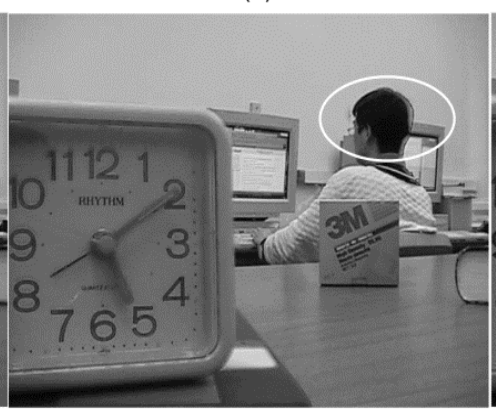

(e)

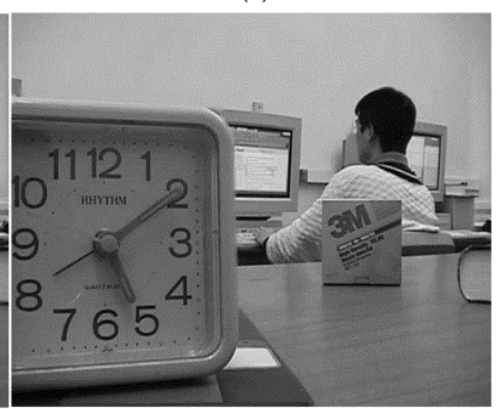

(f)

Figure 4. Fused Images 'Lab' Obtained by DWT (a), SF (b), RPCA (c), PCNN1 (d), PCNN2 (e), the Proposed Method (f) 


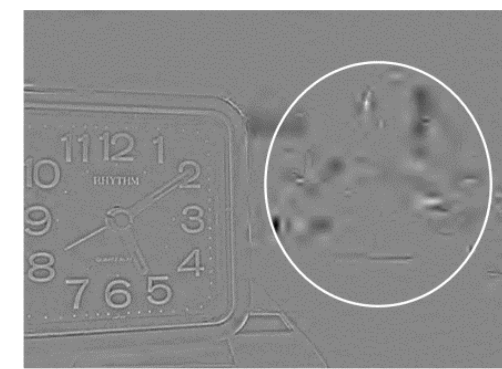

(a)

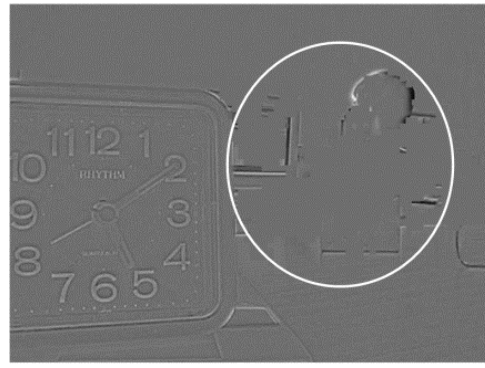

(d)

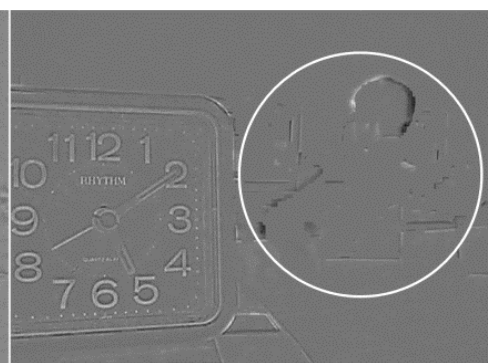

(b)

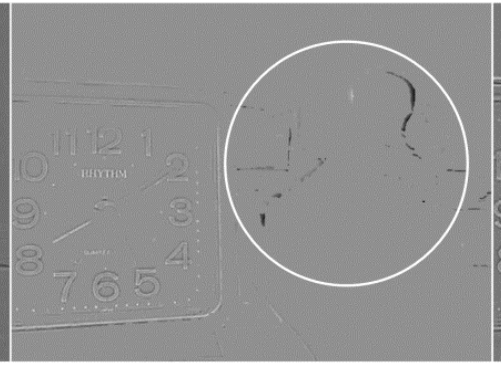

(e)

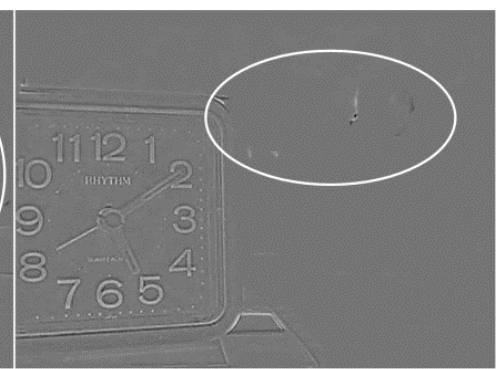

(c)

Figure 5. Difference Images between the Far Focused Source Image 'Lab' and Corresponding Fused Images obtained by DWT (a), SF (b), RPCA (c), PCNN1 (d), PCNN2 (e), the Proposed Method (f)

\subsection{Quantitative Analysis}

For quantitative comparison, the quantitative results in two quality measures are shown in Table 1 . The proposed method gains highest MI and $Q^{A B / F}$ values compared to the other fused methods. The running times are also showed in Table 1. The proposed method still yields longer computational cost than DWT-based fusion method and SFbased fusion method, and the matrix decomposition accounts for the majority of the computational load.

Table 1. Performance of Different Fusion Methods

\begin{tabular}{|c|c|c|c|c|c|c|}
\hline \multirow{2}{*}{ Method } & \multicolumn{3}{|c|}{ Book } & \multicolumn{3}{c|}{ Lab } \\
\cline { 2 - 7 } & MI & $O^{A B / F}$ & Run-time(s) & MI & $O^{A B / F}$ & Run-time(s) \\
\hline DWT & 6.82 & 0.69 & 0.52 & 6.47 & 0.69 & 0.59 \\
\hline SF & 8.41 & 0.70 & 1.04 & 7.94 & 0.72 & 1.03 \\
\hline RPCA & 9.27 & 0.73 & 63.08 & 8.50 & 0.75 & 60.80 \\
\hline PCNN1 & 9.37 & 0.70 & 0.56 & 8.86 & 0.71 & 0.55 \\
\hline PCNN2 & 9.45 & 0.68 & 32.30 & 8.78 & 0.68 & 32.51 \\
\hline Proposed & $\mathbf{9 . 5 7}$ & $\mathbf{0 . 7 5}$ & 1.10 & $\mathbf{8 . 9 2}$ & $\mathbf{0 . 7 7}$ & 1.09 \\
\hline
\end{tabular}

\section{Conclusion and Future Work}

In this paper, a novel pulse coupled neural network based fusion method is proposed. Experimental results demonstrate that the proposed method achieves better fusion results compared to the other existing fusion methods and significantly improves the quality of the fused image. In the future, we will consider optimizing the proposed 
method to reduce the time-consumption and improve the adaptivity of the proposed method.

\section{Acknowledgements}

The work is supported by the National Key Technology Science and Technique Support Program (No. 2013BAH49F03), the National Nature Science Foundation of China (No. 61379010), the Key Technologies R\&D Program of Henan Province (No. 132102210515), the Natural Science Basic Research Plan in Shaanxi Province of China (No. 2012JQ1012).

\section{References}

[1] S. Li, X. Kang, J. Hu and B. Yang, "Image matting for fusion of multi-focus images in dynamic scenes", Information Fusion, vol. 14, (2013).

[2] H. Hariharan, "Extending Depth of Field via Multi-focus Fusion", PhD Thesis, the University of Tennessee, Knoxville, (2011).

[3] R. Eckhorn, H. J. Reitboeck, M. Arndt and P.W. Dicke, "Feature linking via synchronization among distributed assemblies: Simulation of results from cat cortex", Neural Computation, vol. 2, (1990).

[4] R. P. Broussard, S. K. Rogers, M. E. Oxley and G. L. Tarr, "Physiologically motivated image fusion for object detection using a pulse coupled neural network", IEEE Transaction Neural Networks, vol. 10, (1999), pp. 554-563.

[5] J. L. Johnson, H. S. Ranganath, G. Kuntimad and H. J. Caulfield, "Pulse coupled neural networks", Neural Networks and Pattern Recognition, (1999).

[6] Z. B. Wang, Y. D. Ma, F. Y. Cheng and L. Z. Yang, "Review of pulse-coupled neural networks", Image and Vision Computing, vol. 28, no. 1, (2010).

[7] Q. G. Miao and B. S. Wang, "A Novel Adaptive Multi-focus Image Fusion Algorithm Based on PCNN and Sharpness", Proceedings of International Society for Optics and Photonics, Bellingham, USA, (2005) January $17-19$.

[8] W. Huang and Z. L. Jing, "Multi-focus image fusion using pulse coupled neural network", Pattern Recognition Letters, vol. 28, no. 9, (2007).

[9] X. B. Qu, J. W. Yan, H. Z. Xiao and Z. Q. Zhu, "Image Fusion Algorithm Based on Spatial FrequencyMotivated Pulse Coupled Neural Networks in Nonsubsampled Contourlet Transform Domain", Acta Automatica Sinica, vol.34, no. 2, (2008).

[10] E. Candes, X. Li, Y. Ma and J. Wright, "Robust principal component analysis?", Journal of the ACM, vol. 58, no. 3, (2011).

[11] W. Zou, K. Kpalma, Z. Liu and J. Ronsin, "Segmentation Driven Low-rank Matrix Recovery for Saliency Detection", 24th British Machine Vision Conference (BMVC). Bristol, (2013), pp. 1-13.

[12] T. Wan, C. Zhub and Z. Qin, "Multifocus Image Fusion Based on Robust Principal Component Analysis", Pattern Recognition Letters, vol. 34, no. 9, (2013).

[13] Z. Lin, M. Chen and Y. Ma, "The augmented Lagrange multiplier method for exact recovery of corrupted low-rank matrices", UIUC Technical Report UILU-ENG-09-2215, (2010).

[14] W. Huang and Z. Jing, "Evaluation of focus measures in multi-focus image fusion", Pattern Recognition Letters, vol. 28, no. 9, (2007).

[15] Y. Zhang and L. Ge, "Efficient fusion scheme for multi-focus images by using blurring measure", Digital Signal Processing, vol. 19, (2009).

[16] H. Li, B. S. Manjunath and S. K. Mitra, "Multisensor image fusion using the wavelet transform", Graphical Models and Image Processing, vol. 57, (1995).

[17] http://www.ece.lehigh.edu/spcrl, online image database, Accessed (2013) April 17.

[18] http://www.imgfsr.com/sitebuilder/images, online image database, Accessed (2013) April 15.

[19] S. Li, J. T. Kwok and Y. Wang, "Combination of images with diverse focuses using the spatial frequency", Information fusion, vol. 2, no. 3, (2001).

[20] http://www.imagefusion.org/, Image fusion toolbox, Accessed (2013) March 20.

[21] http://perception.csl.illinois.edu/matrix-rank/sample_code.html, RPCA toolbox, Accessed (2013) January 10.

[22] http://quxiaobo.go.8866.org/project/PCNN/PCNN_toolbox.rar, PCNN toolbox, Accessed (2013) January 10.

[23] D. J. C. MacKay, "Information theory, inference and learning algorithms", Cambridge university press, (2003).

[24] C. S. Xydeas and V. Petrovic, "Objective image fusion performance measure”, Electronics Letters, vol. 36, no. $4,(\mathbf{2 0 0 0})$. 


\begin{abstract}
Authors
Yongxin Zhang, currently pursuing a $\mathrm{PhD}$ degree at the School of Information Science and Technology, Northwest University, Xi'an, China. His research interests include image processing and pattern recognition.
\end{abstract}

Li Chen, a professor at the School of Information Science and Technology, Northwest University, Xi'an, China. Her research interests include intelligent information processing, data mining and pattern recognition.

Zhihua Zhao, currently pursuing a PhD degree at the School of Information Science and Technology, Northwest University, Xi'an, China. His research interests include image processing.

Jian Jia, an associate professor at the Department of Mathematics, Northwest University, $\mathrm{Xi}$ 'an, China. His research interests include image restoration, multi-resolution representations and intelligent information processing. 
International Journal of Signal Processing, Image Processing and Pattern Recognition Vol.7, No.3 (2014) 